\title{
Thermal conductivity, electrical resistivity, and dimensionless figure of merit (ZT) determination of thermoelectric materials by impedance spectroscopy up to $250^{\circ} \mathrm{C}$
}

Braulio Beltrán-Pitarch, Jesús Prado-Gonjal, Anthony V. Powell, Pawel Ziolkowski, and Jorge García-Cañadas

Citation: Journal of Applied Physics 124, 025105 (2018); doi: 10.1063/1.5036937

View online: https://doi.org/10.1063/1.5036937

View Table of Contents: http://aip.scitation.org/toc/jap/124/2

Published by the American Institute of Physics

\section{Articles you may be interested in}

Theoretical efficiency of hybrid solar thermoelectric-photovoltaic generators Journal of Applied Physics 124, 024501 (2018); 10.1063/1.5022569

A practical field guide to thermoelectrics: Fundamentals, synthesis, and characterization Applied Physics Reviews 5, 021303 (2018); 10.1063/1.5021094

Influence of convection at outer ceramic surfaces on the characterization of thermoelectric modules by impedance spectroscopy

Journal of Applied Physics 123, 084505 (2018); 10.1063/1.5019881

Phonon anharmonicity in thermoelectric palladium sulfide by Raman spectroscopy

Applied Physics Letters 113, 022105 (2018); 10.1063/1.5041973

Effect of high-pressure torsion on the microstructure and thermoelectric properties of $\mathrm{Fe}_{2} \mathrm{VAI}-$ based compounds Journal of Applied Physics 124, 035106 (2018); 10.1063/1.5034390

Single layer In-O atomic sheets as phonon and electron barriers in $\mathrm{ZnO}-\mathrm{In}_{2} \mathrm{O}_{3}$ natural superlattices: Implications for thermoelectricity

Journal of Applied Physics 124, 025101 (2018); 10.1063/1.5027625

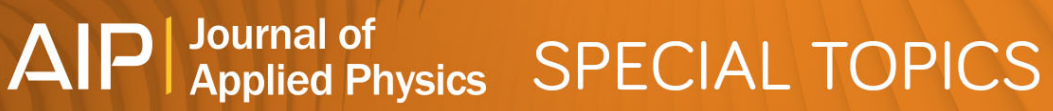

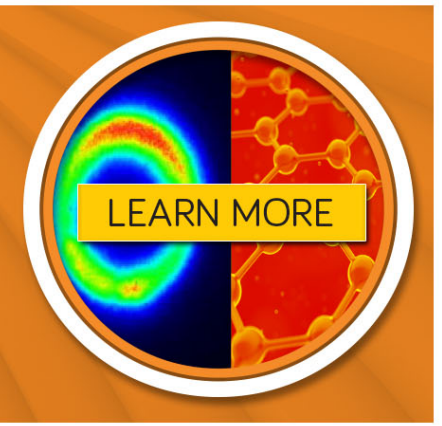




\title{
Thermal conductivity, electrical resistivity, and dimensionless figure of merit (ZT) determination of thermoelectric materials by impedance spectroscopy up to $250^{\circ} \mathrm{C}$
}

\author{
Braulio Beltrán-Pitarch, ${ }^{1}$ Jesús Prado-Gonjal, ${ }^{2}$ Anthony V. Powell, ${ }^{2}$ Pawel Ziolkowski, ${ }^{3}$ \\ and Jorge García-Cañadas ${ }^{1, a)}$ \\ ${ }^{1}$ Department of Industrial Systems Engineering and Design, Universitat Jaume I, Campus del Riu Sec, \\ 12071 Castellón, Spain \\ ${ }^{2}$ Department of Chemistry, University of Reading, RG6 6AD Reading, United Kingdom \\ ${ }^{3}$ Institute of Materials Research, German Aerospace Center, Linder Höhe, 51147 Köln, Germany
}

(Received 19 April 2018; accepted 21 June 2018; published online 13 July 2018)

\begin{abstract}
Impedance spectroscopy has been shown as a promising method to characterize thermoelectric (TE) materials and devices. In particular, the possibility to determine the thermal conductivity $\lambda$, electrical conductivity $\sigma$, and the dimensionless figure of merit $Z T$ of a TE element, if the Seebeck coefficient $S$ is known, has been reported, although so far for a high-performance TE material $\left(\mathrm{Bi}_{2} \mathrm{Te}_{3}\right)$ at room temperature. Here, we demonstrate the capability of this approach at temperatures up to $250^{\circ} \mathrm{C}$ and for a material with modest TE properties. Moreover, we compare the results obtained with values from commercial equipment and quantify the precision and accuracy of the method. This is achieved by measuring the impedance response of a skutterudite material contacted by $\mathrm{Cu}$ contacts. The method shows excellent precision (random errors $<4.5 \%$ for all properties) and very good agreement with the results from commercial equipment $(<4 \%$ for $\lambda$, between $4 \%$ and $6 \%$ for $\sigma$, and $<8 \%$ for ZT), which proves its suitability to accurately characterize bulk TE materials. Especially, the capability to provide $\lambda$ with good accuracy represents a useful alternative to the laser flash method, which typically exhibits higher errors and requires the measurement of additional properties (density and specific heat), which are not necessarily needed to obtain the ZT. Published by AIP Publishing. https://doi.org/10.1063/1.5036937
\end{abstract}

\section{INTRODUCTION}

An efficient use of energy becomes increasingly important. Thermoelectric (TE) devices are considered among other means to improve the energy efficiency of combustion engines or to harvest energy from industrial processes by a conversion of waste heat to electric power. The efficiency of a TE material is related to the dimensionless figure of merit $Z T=\sigma S^{2} T / \lambda$, where $\sigma$ is the electrical conductivity, $S$ is the Seebeck coefficient, $T$ is the absolute temperature, and $\lambda$ is the thermal conductivity. The search for more efficient materials is typically guided by the $Z T$ improvement, which can be obtained by adjustment of the composition, doping, microstructural effects and nano-structuring. ${ }^{1} Z T$ is usually obtained from the independent determination of the three properties that define it $(\sigma, S$, and $\lambda)$. For this reason, TE characterization is a time-consuming task which usually requires several apparatus. Moreover, the determination of the thermal conductivity is especially troublesome, since heat losses are difficult to minimize and high errors are frequently present. The laser flash method $^{2}$ is the most frequently used technique for the thermal conductivity determination, ${ }^{3}$ but it requires the additional measurement of two more properties (density and specific heat), which complicates the TE characterization and introduces measurement uncertainties, especially with respect to the specific heat.

${ }^{\text {a)} E-m a i l: ~ g a r c i a j @ u j i . e s ~}$
Under this scenario, new techniques and methods are highly desired to improve the task of TE characterization by reducing the required efforts, the time, and by improving accuracy.

Impedance spectroscopy has been shown as a promising method to characterize TE materials and devices. ${ }^{4-8}$ This technique has been employed in many fields of research (fuel cells, ${ }^{9}$ supercapacitors, ${ }^{10}$ construction, ${ }^{11}$ corrosion, ${ }^{12}$ photovoltaics, ${ }^{13}$ etc.). Due to this, impedance equipment can be easily found in many research institutions, and highly accurate and reliable apparatus exist. In our previous work, ${ }^{6,14}$ we identified, for a high-performance TE material $\left(\mathrm{Bi}_{2} \mathrm{Te}_{3}\right)$ and at room temperature, the possibility to determine its thermal conductivity, electrical resistivity, and $Z T$, if the Seebeck coefficient is known. However, for materials characterization, this approach has neither been extended to high temperatures nor has been evaluated for lowperformance TE materials. The latter could be troublesome due to the very small impedance signals typically registered (in the $\mathrm{m} \Omega$ range), which might be close to the equipment limitation. ${ }^{14}$ The signal originates from the low Seebeck voltage induced by the Peltier effect when the current is applied. In addition, a quantification of the precision and accuracy of the impedance method to determine the TE properties of bulk materials using this approach has not been previously provided.

In this work, we extend the previously mentioned approach above room temperature (up to $250^{\circ} \mathrm{C}$ ), and 
demonstrate its capability to measure low-performance TE materials. This is achieved using a skutterudite material, which exhibits low $Z T(<0.2)$ around room temperature. The sample is measured in a homemade setup which is adapted to perform measurements in a 4-probe mode. Using experimentally measured values of the Seebeck coefficient from a commercial equipment, the rest of TE properties were determined by the impedance method using a suitable equivalent circuit. Finally, the precision and accuracy of the technique was evaluated by a comparison of the obtained TE properties with results from commercial equipment.

\section{EXPERIMENTAL SETUP}

The homemade setup used for the impedance characterization contains a sample holder suitable for TE materials of bar shape, which is shown in Fig. 1. To perform the measurements, the TE sample is sandwiched between two pieces of copper of same cross-sectional area as the TE material and with $2 \mathrm{~mm}$ thickness. This is required to ensure a homogeneous electrical current at the junctions and a uniform Peltier effect. A very thin layer of $\mathrm{Ga}_{62} \mathrm{In}_{22} \mathrm{Sn}_{16}$ liquid metal (Ref. 14634, Alfa Aesar) was spread homogeneously at the junctions, which were previously polished and cleaned with acetone to provide a good thermal and electrical contact. For the same reason, it is important that the $\mathrm{Cu}$ and TE material surfaces brought into contact are as flat as possible. Two very thin copper wires ( $15 \mu \mathrm{m}$ diameter, Alfa Aesar) were inserted in both junctions for the measurement of the voltage difference across the TE sample (see inset of Fig. 1). The very thin diameter minimizes the heat losses by conduction through the wires, and also allows the wires to be inserted at the junctions.

Once assembled, the sample is clamped at the sample holder by two sharpened stainless steel screws, which act as probes to supply the current flow. These two screws are screwed by nuts at holed ceramics (Macor, Corning) which provide electrical insulation. The stainless steel screws are connected to thick copper wires insulated by ceramic beads (see Fig. 1). Stainless steel screws were chosen due to their

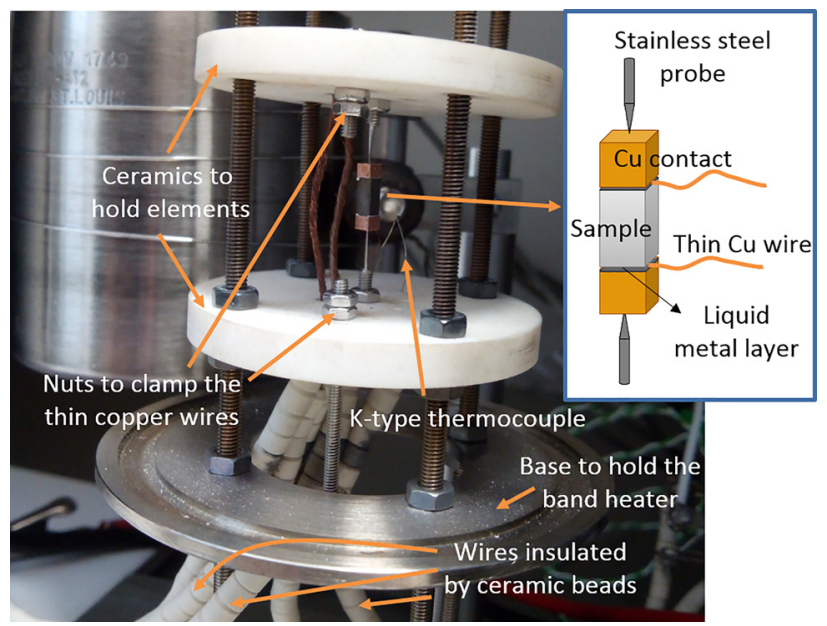

FIG. 1. Photograph of the sample holder employed for the impedance characterization of thermoelectric materials. The inset describes schematically how the sample is contacted. low thermal conductivity $[\approx 14 \mathrm{~W} /(\mathrm{Km})]$, which reduces heat losses by conduction. They were also sharpened for the same purpose. The very thin copper wires that measure the potential difference are clamped at the sample holder by two nuts screwed with stainless steel screws, which are held by the ceramic plates (see Fig. 1). These screws are also connected to thick copper wires insulated by ceramic beads. The bottom holed ceramic disc is fixed at four threaded studs by nuts, while the top ceramic is free to move to be able to allocate samples of different lengths, and additionally provide certain pressure to the contacts. A stainless steel base is also held by nuts at the studs. This base is used to hold a band heater (Ref. MB2E2JN1-B12, Watlow) which surrounds the sample holder and is used to provide different ambient temperatures. The ambient temperature is measured by a K-type thermocouple (RS) placed close to the TE sample (see Fig. 1 ), whose temperature is controlled by a temperature controller (Watlow EZ Zone PM) which powers the heater.

All the impedance measurements were performed inside a stainless steel vacuum chamber at pressure values $<10^{-4}$ mbar in order to eliminate convection heat losses. In addition, the metallic vacuum chamber also serves as a Faraday cage, which reduces electromagnetic noise during the measurements. The TE sample used in this study was a tetragonal and isotropic $n$-type skutterudite $\left(\mathrm{CoSb}_{2.75} \mathrm{Sn}_{0.05} \mathrm{Te}_{0.20}\right)$, which was cut with a diamond saw of $0.3 \mathrm{~mm}$ diameter from a disc pellet. A suitable cutting is important to obtain a crack free sample of highly uniform cross-sectional area. The crosssectional area of the sample was $2.30 \mathrm{~mm} \times 2.11 \mathrm{~mm}$ and its length was $5.01 \mathrm{~mm}$. The skutterudite sample was characterized employing commercial equipment before the impedance measurements. This charaterisation with commercial equipment was performed to the original disc pellet. A Linseis LSR-3 equipment was used to determine the electrical resistivity and the Seebeck coefficient. For the thermal conductivity, a Netzsch LFA 447 laser flash apparatus was employed. The specific heat of the sample was determined using the same equipment via a comparative method with a Pyroceram reference sample. The density of the sample, which is also required for the determination of the thermal conductivity by the laser flash method, was measured using an Archimedes balance.

A PGSTAT30 potentiostat (Metrohm Autolab B.V.) equipped with a FRA2 impedance module and a BOOSTER10A, which amplifies the maximum current of the equipment up to $10 \mathrm{~A}$, was used to perform the impedance spectroscopy measurements. Although such large currents were not reached, the booster is used in order to reduce a systematic jump in the real impedance of $\approx 70 \mu \Omega$ produced due to a change in the gain of the equipment, which occurs at frequencies around $25 \mathrm{~Hz}$ (see Fig. S1). This jump can be significantly reduced if measurements are performed in the largest possible current range. At each temperature, the impedance measurement was conducted in 40 logarithmically distributed frequency steps between $5 \mathrm{mHz}$ and $10 \mathrm{kHz}$. An AC current without steady component $\left(I_{\mathrm{DC}}=0\right.$ A) was employed using a maximum integration time of $2 \mathrm{~s}$ and 2 minimum integration cycles. The AC current amplitude to be used needs to be optimized, since significant differences in the spectra can be observed when this parameter 
is varied (see Fig. S2). This optimization is described in Sec. III B. Nova 1.11 software was used to control the potentiostat and record the experimental signals. Experimental impedance spectra were fitted to equivalent circuits using Zview software.

\section{RESULTS AND DISCUSSION}

\section{A. The equivalent circuit}

In order to extract the properties of interest from the impedance spectra, the experimental results are typically fitted to a suitable theoretical model (equivalent circuit), which should describe the physics of the device. The equivalent circuit corresponding to the case of a TE sample contacted by two metallic contacts has been previously reported, ${ }^{6}$ and consists of an ohmic resistance $R_{\Omega}$ connected in series with the parallel combination of two Warburg elements: a constant temperature Warburg impedance $Z_{W C T}$, which relates to the properties of the TE sample, and an adiabatic Warburg impedance $Z_{W a}$, which is described by $S$ and the properties of the metallic contact material. These elements are defined as follows: ${ }^{6,15}$

$$
\begin{gathered}
R_{\Omega}=\frac{\rho_{T E} L_{T E}}{A} \\
Z_{W C T}=R_{T E}\left(\frac{j \omega}{\omega_{T E}}\right)^{-0.5} \tanh \left[\left(\frac{j \omega}{\omega_{T E}}\right)^{0.5}\right], \\
Z_{W a}=R_{C}\left(\frac{j \omega}{\omega_{C}}\right)^{-0.5} \tanh \left[\left(\frac{j \omega}{\omega_{C}}\right)^{0.5}\right],
\end{gathered}
$$

where $\rho_{T E}, L_{T E}$, and $A$ are the electrical resistivity, length, and cross-sectional area of the TE material, respectively. $R_{T E}$ is the TE resistance given by

$$
R_{T E}=\frac{S^{2} T L_{T E}}{\lambda_{T E} A},
$$

where $T$ is the absolute ambient temperature and $\lambda_{T E}$ is the thermal conductivity of the TE material. $j=(-1)^{0.5}, \omega$ is the angular frequency, and $\omega_{T E}$ is the characteristic angular frequency of thermal diffusion in the TE sample $\left(\omega_{T E}=\alpha_{T E} /\right.$ $\left(L_{T E} / 2\right)^{2} ; \alpha_{T E}$ being the thermal diffusivity of the TE material). $R_{C}$ is the TE resistance induced by the metallic contact $(\mathrm{Cu}$ pieces) given by

$$
R_{C}=2 \frac{S^{2} T L_{C}}{\lambda_{C} A},
$$

where $\lambda_{C}$ and $L_{C}$ are the thermal conductivity and length of the metallic contact material, respectively. Finally, $\omega_{C}$ is the characteristic angular frequency of thermal diffusion in the contact $\left[\omega_{C}=\alpha_{C} /\left(L_{C}\right)^{2} ; \alpha_{C}\right.$ being the thermal diffusivity of the contact].

It should be noted that due to the high thermal conductivity of copper $[\approx 400 \mathrm{~W} /(\mathrm{Km})], R_{C}$ has a very low value $(\approx 15$ $\mu \Omega$ at room $T$ ). For this reason, the slope- 1 part of the $Z_{W a}$ element is not clearly observed experimentally and this element takes the form of a capacitor, with its impedance function described by $Z_{C c}=1 /(j \omega C c)$, being $C c=\left(R_{C} \omega_{C}\right)^{-1}{ }^{6}$ This equivalent circuit, which was used to perform the fittings to the experimental impedance results, is shown in the inset of Fig. 2(a). It describes a semicircle in the complex plane (Nyquist plot), where the ohmic resistance and $R_{T E}$ can be clearly identified as the high frequency (left side) intercept with the real axis and the diameter of the semicircle, respectively. ${ }^{6}$

From the fittings, $R_{\Omega}, R_{T E}, \omega_{T E}$, and $C_{C}$ can be obtained. Hence, using Eqs. (1) and (4), the electrical resistivity, thermal conductivity (if $S$ is known), and $Z T$ of the TE material can be obtained. From Eq. (1)

$$
\rho_{T E}=\frac{R_{\Omega} A}{L_{T E}} .
$$

From Eq. (4)

$$
\lambda_{T E}=\frac{S^{2} T L_{T E}}{R_{T E} A} .
$$

Combining Eqs. (1) and (4)

$$
Z T=\frac{R_{T E}}{R_{\Omega}} .
$$

\section{B. Current amplitude optimization}

Before characterizing the skutterudite sample at the different temperatures, it is important to identify the suitable current amplitude to be used during the impedance measurements. Figure S2 shows impedance spectra performed at an
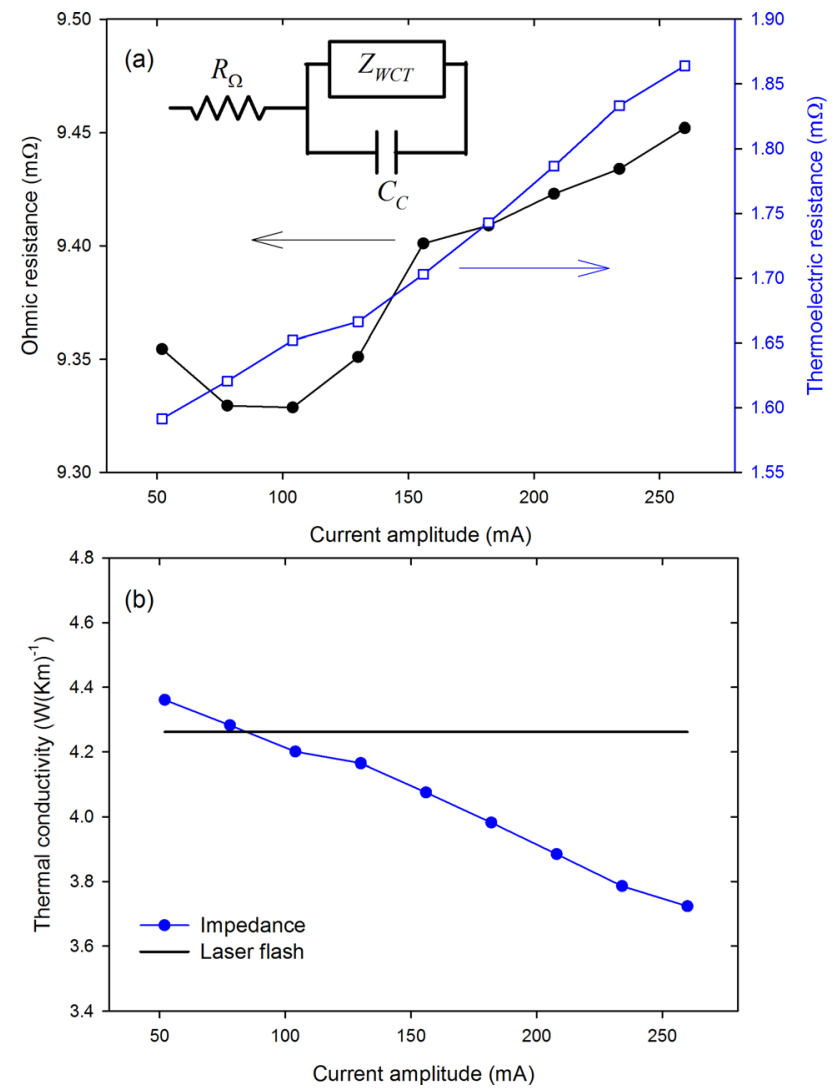

FIG. 2. Variation of (a) the ohmic, the thermoelectric resistance, and (b) the extracted thermal conductivity, with different current amplitudes employed in the impedance experiments at $50{ }^{\circ} \mathrm{C}$ of Fig. S2. The inset in (a) shows the equivalent circuit used for the fittings. 
ambient temperature of $50^{\circ} \mathrm{C}$ for different current amplitudes $I_{a c}$. It can be observed that the spectra vary with the current amplitude, probably due to the existence of Joule effect and/or the dependence of the TE properties on temperature. From Fig. S2(a), which shows the experiment at the lowest current amplitude $(52 \mathrm{~mA})$, it can be observed that the spectrum is somewhat noisy, due to the existence of several points which deviate from the shape of a semicircle. The noise is reduced when $78 \mathrm{~mA}$ amplitude is used, yielding a better correspondence to the semicircle characteristic although some points still deviate in the higher frequency range [see inset of Fig. S2(b)]. At amplitudes of $104 \mathrm{~mA}$ and above, the noise becomes negligible, consequently, this amplitude is considered as optimum, since it provides a sufficient Peltier effect to obtain a clear TE signal in the impedance spectrum while a minimal Joule heat liberation is ensured at the same time. This amplitude corresponds to a Peltier heat power per unit area $\left(S T I_{a c} / A\right)$ generated at the junctions of $1000 \mathrm{~W} / \mathrm{m}^{2}$. Using this value as reference, the optimum current amplitude to be employed at the different temperatures is calculated by $I_{a c}=\left(1000 \mathrm{~W} / \mathrm{m}^{2}\right) A /(S T)$, obtaining values of $84,69,58$, and $49 \mathrm{~mA}$ for the ambient temperatures of $100,150,200$, and $250^{\circ} \mathrm{C}$, respectively.
In Fig. 2(a), the values for $R_{\Omega}$ and $R_{T E}$ corresponding to the experiments of Fig. S2 are quantified. It can be observed that the ohmic resistance varies randomly at the lower current amplitudes and at values higher than $150 \mathrm{~mA}$, it starts to increase monotonically. This increase could be due to an increase in the electrical resistivity of the TE sample induced by a temperature rise due to the heating by Joule effect, which becomes more intense as $I_{a c}$ is increased. On the other hand, it can be observed from Fig. S2 that the semicircle in the impedance spectra widens as the current amplitude is increased, which translates into an increase in $R_{T E}$ with $I_{a c}$, as it is shown in Fig. 2(a). This increase could be also due to the Joule effect and the increase in the electrical resistivity of the skutterudite material with temperature, but, in addition, an increase in the average Seebeck coefficient, a decrease in the thermal conductivity, and the initial sample $T$, can contribute [see Eq. (4)]. Figure 2(b) shows the calculated values of the thermal conductivity from $R_{T E}$ and the Seebeck coefficient values using Eq. (7). The latter are provided by measurements from the commercial equipment [see inset of Fig. 3(b)]. The thermal conductivity value measured by the laser flash equipment is also shown in Fig. 2(b) as reference. It can be observed that good agreement is found
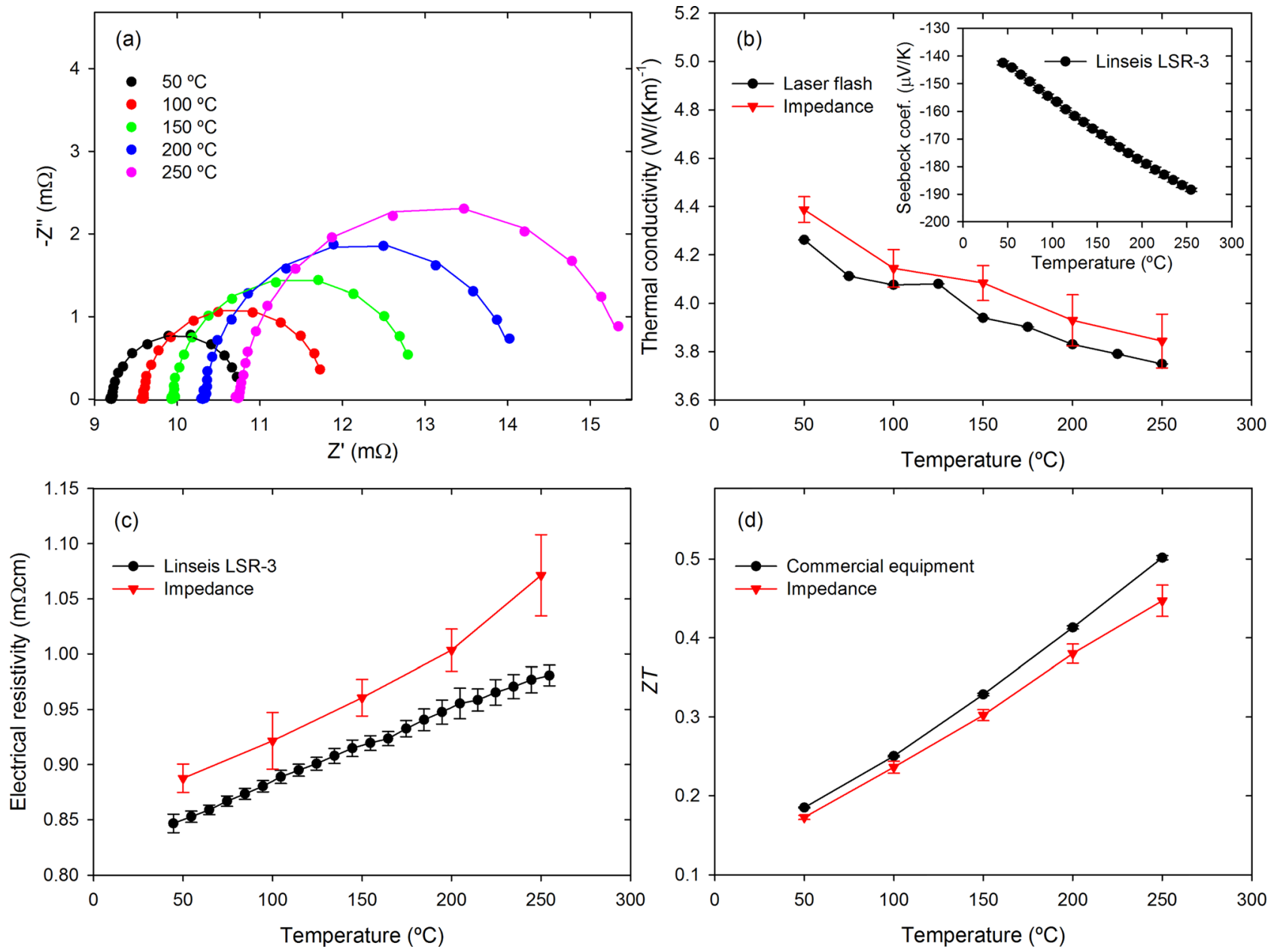

FIG. 3. (a) Impedance spectroscopy measurements at different temperatures from one of the five measurement cycles performed. The lines represent the fittings to the experimental values. (b) Thermal conductivity, (c) electrical resistivity and (d) ZT values extracted from the impedance method and compared with results from different commercial equipment. The inset in (b) shows the Seebeck coefficient measured by the Linseis LSR-3 equipment, which is required to obtain the thermal conductivity by the impedance method. The error bars account for the total combined random errors, excluding the contribution from the specific heat for the laser flash case. 
for the lower amplitudes $(<110 \mathrm{~mA})$, and higher $I_{a c}$ values lead to significant deviations. The previously optimized value of $104 \mathrm{~mA}$ (corresponding to $1000 \mathrm{~W} / \mathrm{m}^{2}$ Peltier heat power per unit area) lies in the low current amplitude range where the agreement with $\lambda_{T E}$ is good and the $R_{\Omega}$ does not tend to increase, which proves its validity.

We also evaluated the effect of the variation of the current amplitude in the impedance spectra using a $2.08 \mathrm{~mm}$ $\times 2.01 \mathrm{~mm} \times 8.00 \mathrm{~mm} \mathrm{Fe}{ }_{0.95} \mathrm{Co}_{0.05} \mathrm{Si}_{2}$ sample, ${ }^{16}$ whose electrical resistivity is around 10 times higher than that for the skutterudite material and, moreover, unlike the skutterudite material, it decreases with temperature [see Fig. S3(a)]. In this sample, the Joule effect is expected to be more prominent. Impedance spectroscopy measurements were performed on this material at room temperature and under ambient air conditions (no vacuum) in the $20 \mathrm{kHz}-10 \mathrm{mHz}$ range and employing different current amplitudes. The obtained results can be seen in Fig. S3(b). It can be observed that, unlike the case of the skutterudite, a shift of the real part of the impedance signal towards lower values is produced, which is more intense at higher current amplitudes. An explanation of this behavior is again possible by the existence of Joule effect and the connected increase in temperature which decreases the electrical resistivity of this material, and hence its ohmic resistance, which yields a shift of the real impedance towards lower values. On the other hand, a decrease in $R_{T E}$ is observed [see inset of Fig. S3(b)], which could be due to the reasons mentioned above but now with a more dominant contribution from the decrease in the electrical resistivity with temperature. In this case, it is also important to optimize the current amplitude in order to minimize the observed shifts (Joule effect).

\section{Characterization by the impedance method}

Using the previously optimized current amplitudes, the skutterudite sample was characterized by impedance spectroscopy at different temperatures in the $50-250^{\circ} \mathrm{C}$ range. Five measurement cycles from 50 to $250^{\circ} \mathrm{C}$ were measured. Each cycle was initiated with remade contacts. Figure 3(a) shows the impedance spectra obtained for one of these cycles. All the spectra show unnoisy measurements and an excellent fitting (solid lines) to the equivalent circuit of Fig. 2(a). Fitting error values $<1 \%$ were obtained for $R_{\Omega}$ and $R_{T E}$ in all cases. It can be observed that even for the spectrum at the lowest temperature, the impedance response is clearly observed. At this temperature, the skutterudite exhibits a lower performance and the equipment is still able to precisely record points which are separated by $\approx 0.1 \mathrm{~m} \Omega$, which demonstrates the capability of this technique to measure materials with modest TE properties.

The TE properties of the skutterudite material were extracted from the average value of the five fitting results of each parameter $\left(R_{\Omega}\right.$ and $\left.R_{T E}\right)$ at each temperature using Eqs. (6) to (8). Figures 3(b)-3(d) show the thermal conductivity, electrical resistivity, and the dimensionless figure of merit ZT obtained from the impedance spectroscopy method, respectively, which are compared with the measurements from commercial equipment. All the properties show a good agreement and reproduce the trends found in the commercial equipment measurements, except the point at the highest temperature $\left(250^{\circ} \mathrm{C}\right)$ from the electrical resistivity. This deviation is attributed to changes experienced by the liquid metal layer employed at the junctions, which tends to solidify at these higher temperatures, and even remains solid when the temperature returns to room values. This introduces a somewhat larger contact resistance which becomes no longer negligible. It should be noted that the very thin $\mathrm{Cu}$ wires which measure the voltage difference are embedded in the junctions (see Fig. 1), and hence are in contact with the liquid metal material, which can contribute to the measured resistance if its influence is not kept low. This fact also limits the maximum temperature of the method, since the rest of the elements of the setup can stand for much higher temperature values, so a most suitable solder or liquid metal could increase the capability of the method to measure at higher temperatures.

\section{Precision and accuracy evaluation}

In order to evaluate the precision and accuracy of the impedance method, random and systematic errors, respectively, were calculated for the thermal conductivity, electrical resistivity and $Z T$. The total combined random errors $u_{c}$ of each parameter were calculated using ${ }^{17}$

$$
u_{c}^{2}=\sum_{i=1}^{N}\left(\frac{\partial f}{\partial x_{i}}\right)^{2} u^{2}\left(x_{i}\right),
$$

with $f$ being each of the TE properties $\left(\rho_{T E}, \lambda_{T E}\right.$, or ZT), and $x_{i}$ being each of the parameters required for the determination of the corresponding TE property with its associated error $u$. The random errors for the thermal conductivity were calculated taking into account (i) the standard deviation from three measurements performed using the commercial equipment for the Seebeck coefficient, (ii) the uncertainty of the thermocouple $\left[u(T)=1^{\circ} \mathrm{C}\right]$, (iii) the uncertainty in the length of the sample which was measured using a caliber $\left[u\left(L_{T E}\right)=0.005 \mathrm{~mm}\right]$, (iv) the uncertainty in the area of the sample, and (v) the standard deviation of the five measurements at each temperature to obtain the average value of $R_{T E}$. The contribution from the fitting errors in $R_{T E}$ (which were $<1 \%$ ) was neglected since it was negligible in comparison with the standard deviation. From Table S1, which shows all these contributions, it can be observed that the contributions from the Seebeck coefficient and $R_{T E}$ are the most significant, being the rest negligible.

The random errors for the electrical resistivity were calculated taking into account (i) the uncertainty in the length of the sample, (ii) the uncertainty in the area, and (iii) the standard deviation from the five measurements at each temperature to obtain the average $R_{\Omega}$. It should be noticed that the latter contribution is the most significant, as shown in Table S1. As occurred for $R_{T E}$, the contribution of the fitting errors $(<1 \%)$ for $R_{\Omega}$ was neglected. Finally, the random errors for $Z T$ were calculated from the contributions of the standard deviations of both $R_{\Omega}$ and $R_{T E}$. In this case, both show similar contributions (see Table S1). The error bars 
shown in Fig. 3 correspond to the calculated combined random errors. In the case of results from the Seebeck coefficient and the electrical resistivity using commercial equipment, the random errors were obtained from the standard deviations of three consecutive measurements performed at each temperature. The combined random error from the thermal conductivity determined by the laser flash method was calculated using Eq. (9) taking into account the standard deviation obtained from three consecutive measurements of the thermal diffusivity and the density. The contribution from the specific heat was not available. Systematic errors $u_{s}$ were calculated for the TE properties considering as true values the results obtained from the commercial equipment. It should be noted that a more rigorous calculation should be performed using standard reference materials (SRM), however, there are no SRM available which could provide simultaneously the three properties measured in this study. ${ }^{3}$

Table I shows the average values of each TE property with their associated random, systematic and total errors $u_{T}$, the latter obtained as $u_{T}=\left(u_{c}{ }^{2}+u_{s}^{2}\right)^{0.5}$. Random errors $<3 \%$ are obtained for $\lambda_{T E}$ and $\rho_{T E}$, except at $250{ }^{\circ} \mathrm{C}$ for the latter, which are somewhat higher due to the reasons previously mentioned. These low values of the random errors demonstrate the excellent precision of the method. For the case of $Z T$, the precision is also excellent, with random errors $\approx 3 \%$, except for the case at $250^{\circ} \mathrm{C}$ due to the higher error in $\rho_{T E}$ at this temperature.

The systematic errors (below $250{ }^{\circ} \mathrm{C}$ ) are $<4 \%$, between $4 \%$ and $6 \%$, and $<8 \%$, for $\lambda_{T E}, \rho_{T E}$, and $Z T$, respectively (see Table I), which demonstrates a good agreement with the characterization performed by commercial equipment. The total errors, found from the contribution of the random and systematic errors, are (excluding the case at $250{ }^{\circ} \mathrm{C}$ ) $\approx 4 \%$, between $4.3 \%$ and $6.2 \%$, and $<9 \%$, for $\lambda_{T E}, \rho_{T E}$ and $Z T$, respectively (see Table I), which proves the suitability to accurately characterize bulk TE materials by the impedance method. Especially, the capability to determine the thermal conductivity with excellent precision and accuracy is remarkable, since it represents an appropriate alternative to the laser flash method, which typically exhibits higher errors and requires the measurement of the density and the specific heat, which are not needed to obtain the ZT. It should be noted that, as we mentioned above, the error bars from the laser flash results in Fig. 3(b) do not include the error contribution from the specific heat, which is usually between $\pm 4 \%$ but can show occasionally much higher variations as shown in a previously conducted round robin campaign. ${ }^{18}$

\section{CONCLUSIONS}

The possibility to determine the electrical resistivity, thermal conductivity (if the Seebeck coefficient is known), and the dimensionless figure of merit $Z T$ of a bulk TE material by impedance spectroscopy has been demonstrated for a low-performance TE material up to $250^{\circ} \mathrm{C}$. A new setup was developed to measure TE materials in a 4-probe mode with the possibility of varying the ambient temperature. A skutterudite material, which shows low-performance at room temperature, was characterized by the impedance method. A clear impedance signal and suitable characterization were obtained even at the lowest temperature, which demonstrates the capability of the method to test low-ZT materials. All the TE properties of the skutterudite sample were determined by fittings performed to the experimental impedance spectra employing a suitable equivalent circuit. It was found to be important to optimize the AC current amplitude to employ in the impedance experiments, since significant variations in the impedance spectra can occur, probably due to the Joule effect and/or the dependence of the thermoelectric properties with temperature. Random errors were calculated by performing five measurements at each temperature with remade contacts, showing an excellent precision of the method (random errors $<4.5 \%$ for all properties). Systematic errors were also determined by comparison with measurements of the sample using commercial equipment, resulting in values $<4 \%$, between $4 \%$ and $6 \%$, and $<8 \%$, for $\lambda_{T E}, \rho_{T E}$ and $Z T$, respectively, which proves the good accuracy of the method.

TABLE I. Average values with their associated random, systematic and total errors of the thermoelectric properties of a skutterudite sample obtained by the impedance spectroscopy method.

\begin{tabular}{|c|c|c|c|c|c|}
\hline & Temperature $\left({ }^{\circ} \mathrm{C}\right)$ & Mean value & Systematic error $(\%)$ & Random error (\%) & Total error $(\%)$ \\
\hline \multirow[t]{5}{*}{ Thermal conductivity $\left(\lambda_{T E}\right)$} & 50 & $4.39 \mathrm{~W} /(\mathrm{Km})$ & 2.95 & 1.21 & 3.19 \\
\hline & 100 & $4.14 \mathrm{~W} /(\mathrm{Km})$ & 1.69 & 1.88 & 2.53 \\
\hline & 150 & $4.08 \mathrm{~W} /(\mathrm{Km})$ & 3.64 & 1.76 & 4.04 \\
\hline & 200 & $3.93 \mathrm{~W} /(\mathrm{Km})$ & 2.60 & 2.69 & 3.74 \\
\hline & 250 & $3.84 \mathrm{~W} /(\mathrm{Km})$ & 2.54 & 2.90 & 3.85 \\
\hline \multirow[t]{5}{*}{ Electrical resistivity $\left(\rho_{T E}\right)$} & 50 & $0.89 \mathrm{~m} \Omega \mathrm{cm}$ & 4.08 & 1.45 & 4.33 \\
\hline & 100 & $0.92 \mathrm{~m} \Omega \mathrm{cm}$ & 4.20 & 2.78 & 5.04 \\
\hline & 150 & $0.96 \mathrm{~m} \Omega \mathrm{cm}$ & 4.87 & 1.72 & 5.17 \\
\hline & 200 & $1.00 \mathrm{~m} \Omega \mathrm{cm}$ & 5.90 & 1.90 & 6.20 \\
\hline & 250 & $1.07 \mathrm{~m} \Omega \mathrm{cm}$ & 9.41 & 3.43 & 10.02 \\
\hline \multirow[t]{5}{*}{ Figure of merit $(Z T)$} & 50 & 0.173 & 6.67 & 1.59 & 6.86 \\
\hline & 100 & 0.236 & 5.62 & 3.22 & 6.48 \\
\hline & 150 & 0.302 & 7.99 & 2.30 & 8.32 \\
\hline & 200 & 0.380 & 7.97 & 3.18 & 8.58 \\
\hline & 250 & 0.447 & 10.87 & 4.42 & 11.73 \\
\hline
\end{tabular}


It is especially remarkable the excellent results found for the characterization of the thermal conductivity, which establishes the impedance method as an alternative approach to the laser flash method, which typically exhibits higher errors and requires additional measurements (density and specific heat), which are not needed to obtain the $Z T$ and which are not necessary in the impedance approach.

\section{SUPPLEMENTARY MATERIAL}

See supplementary material for impedance results using different equipment configurations, impedance spectra of the skutterudite at different current amplitudes, impedance spectra of the $\mathrm{Fe}_{0.95} \mathrm{Co}_{0.05} \mathrm{Si}_{2}$ sample at different current amplitudes, and the contributions to the calculated combined random errors.

\section{ACKNOWLEDGMENTS}

B.B.P. and J.G.C. acknowledge financial support from the Spanish Agencia Estatal de Investigación under the Ramón y Cajal program (RYC-2013-13970), from the Universitat Jaume I under the Project No. UJI-A2016-08, and the technical support of Raquel Oliver Valls and José Ortega Herreros. A.V.P. and J.P.G. wish to thank the UK Engineering and Physical Sciences Research Council (EP/ K019767/1) for financial support.
${ }^{1}$ J. R. Sootsman, D. Y. Chung, and M. G. Kanatzidis, Angew. Chem. Int. Ed. 48, 8616 (2009).

${ }^{2}$ W. J. Parker, R. J. Jenkins, C. P. Butler, and G. L. Abbott, J. Appl. Phys. 32, 1679 (1961).

${ }^{3}$ K. A. Borup, J. de Boor, H. Wang, F. Drymiotis, F. Gascoin, X. Shi, L. Chen, M. I. Fedorov, E. Müller, B. B. Iversen, and G. J. Snyder, Energy Environ. Sci. 8, 423 (2015)

${ }^{4}$ A. D. Downey, T. P. Hogan, and B. Cook, Rev. Sci. Instrum. 78, 93904 (2007).

${ }^{5}$ A. De Marchi and V. Giaretto, Rev. Sci. Instrum. 82, 104904 (2011).

${ }^{6}$ J. García-Cañadas and G. Min, J. Appl. Phys. 116, 174510 (2014).

${ }^{7}$ C.-Y. Yoo, Y. Kim, J. Hwang, H. Yoon, B. J. Cho, G. Min, and S. H. Park, Energy 152, 834 (2017).

${ }^{8}$ Y. Apertet and H. Ouerdane, Energy Convers. Manage. 149, 564 (2017).

${ }^{9}$ X. Z. Yuan, H. J. Wang, J. C. Sun, and J. J. Zhang, Int. J. Hydrogen Energy 32, 4365 (2007).

${ }^{10}$ R. Kotz, M. Hahn, and R. Gallay, J. Power Sources 154, 550 (2006).

${ }^{11}$ S. A. Grammatikos, R. J. Ball, M. Evernden, and R. G. Jones, Compos. Part A: Appl. Sci. Manuf. 105, 108-117 (2018).

${ }^{12}$ G. W. Walter, Corros. Sci. 26, 681 (1986).

${ }^{13}$ F. Fabregat-Santiago, G. Garcia-Belmonte, I. Mora-Sero, and J. Bisquert, Phys. Chem. Chem. Phys. 13, 9083 (2011).

${ }^{14}$ J. García-Cañadas and G. Min, Thermoelectric Material Devices (Royal Society of Chemistry, Cambridge, 2016), pp. 133-155.

${ }^{15}$ J. García-Cañadas and G. Min, AIP Adv. 6, 035008 (2016).

${ }^{16}$ P. Ziolkowski, C. Stiewe, J. de Boor, I. Druschke, K. Zabrocki, F. Edler, S. Haupt, J. König, and E. Mueller, J. Electron. Mater. 46, 51 (2017).

${ }^{17}$ Evaluation of Measurement Data - GUM: Guide to the Expression of Uncertainty in Measurement (1995), https://www.bipm.org/en/ publications/guides/gum.html.

${ }^{18}$ H. Wang, W. D. Porter, H. Bottner, J. Konig, L. D. Chen, S. Q. Bai, T. M. Tritt, A. Mayolet, J. Senawiratne, C. Smith, F. Harris, P. Gilbert, J. Sharp, J. Lo, H. Kleinke, and L. Kiss, J. Electron. Mater. 42, 1073 (2013). 